\title{
DIELECTRONIC RECOMBINATION IN PHOTOIONIZED GAS: THE IMPORTANCE OF FINE-STRUCTURE CORE EXCITATIONS
}

\author{
D. W. Savin, ${ }^{1,2}$ T. Bartsch, ${ }^{3}$ M. H. Chen, ${ }^{4}$ S. M. Kahn, ${ }^{1,2}$ D. A. Liedahl, ${ }^{4}$ J. Linkemann, ${ }^{5}$ \\ A. Müller, ${ }^{3}$ S. Schippers, ${ }^{3}$ M. Schmitt, ${ }^{5}$ D. Schwalm, ${ }^{5}$ And A. Wolf ${ }^{5}$ \\ Received 1997 July 9; accepted 1997 August 28; published 1997 September 23
}

\begin{abstract}
At the low electron temperatures existing in photoionized gases with cosmic abundances, dielectronic recombination (DR) proceeds primarily via $n l_{j} \rightarrow n l_{j^{\prime}}^{\prime}$ excitations of core electrons $(\Delta n=0 \mathrm{DR})$. At these temperatures, the dominant DR channel often involves $2 p_{1 / 2} \rightarrow 2 p_{3 / 2}$ fine-structure core excitations, which are not included in $L S$-coupling calculations or the Burgess formula. Using the heavy-ion storage ring at the Max-Planck-Institut für Kernphysik in Heidelberg, Germany, we have verified experimentally for Fe XVIII that DR proceeding via this channel can be significant in relation to other recombination rates, especially at the low temperatures characteristic of photoionized gases. At temperatures in photoionized gases near where Fe XVIII peaks in fractional abundance, our measured Fe XVIII to Fe XVII $\Delta n=0$ DR rate coefficient is a factor of $\sim 2$ larger than predicted by existing theoretical calculations. We provide a fit to our measured rate coefficient for ionization equilibrium models. We have carried out new fully relativistic calculations using intermediate coupling, which include the $2 p_{1 / 2} \rightarrow 2 p_{3 / 2}$ channel and agree to within $\sim 30 \%$ with our measurements. DR via the $2 p_{1 / 2} \rightarrow 2 p_{3 / 2}$ channel may also have spectroscopic implications, providing unique spectral signatures at soft X-ray wavelengths that could provide good electron temperature diagnostics.
\end{abstract}

Subject headings: atomic data — atomic processes — line: formation - X-rays: general

\section{INTRODUCTION}

The ionization and heating of the media surrounding accretion-powered compact sources, such as cataclysmic variables, $\mathrm{X}$-ray binaries, and active galactic nuclei, are dominated by photoionization (see Kahn \& Liedahl 1995, and references therein). Theoretical models of photoionized gases show that the ionization structure is determined by photoionization balanced by radiative recombination (RR) and dielectronic recombination (DR). Under these conditions the local ionization balance of a plasma is determined by the ionization parameter $\xi=L /\left(n_{e} r^{2}\right)$, where $L$ is the luminosity of the X-ray source, $r$ is the distance from the source, and $n_{e}$ is the electron density (Tarter, Tucker, \& Salpeter 1969). The electron temperature at which the fractional abundance of a given ion peaks (Kallman \& McCray 1982; Kallman et al. 1996) is far below the temperature where the ion would exist in coronal equilibrium (Arnaud \& Rothenflug 1985, hereafter AR85; Arnaud \& Raymond 1992, hereafter AR92). As a result, X-ray line emission is produced by RR and DR and not by electron impact excitation (Liedahl et al. 1990; Kallman et al. 1996). Also, the radiative recombination continuum (RRC) of an ion is predicted to appear as a distinct narrow feature just above the ionization threshold of the ion (Hatchett, Buff, \& McCray 1976).

With the improved spectral resolution offered by the SIS detectors of ASCA (Tanaka, Inoue, \& Holt 1994), it has become possible to observe some of these unique properties of photoionized gases. From ASCA observations, Angelini et al. (1995) have identified the Ne X RRC in SIS spectra of the low-

\footnotetext{
${ }^{1}$ Department of Physics and Astronomy and Space Sciences Laboratory, University of California, Berkeley, CA 94720.

${ }^{2}$ Present address: Department of Physics and Columbia Astrophysics Laboratory, Columbia University, New York, NY 10027.

${ }^{3}$ Institut für Kernphysik, Strahlenzentrum der Justus-Liebig-Universität, D35392, Giessen, Germany.

${ }^{4}$ Lawrence Livermore National Laboratory, Livermore, CA 94550.

${ }^{5}$ Max-Planck-Institut für Kernphysik and Physikalisches Institut der Universität, Heidelberg, D-69117 Heidelberg, Germany.
}

mass X-ray pulsar 4U 1626-67, and Liedahl \& Paerels (1996) have identified the Mg XII, Si XIV, and S XVI RRC in spectra of the X-ray binary Cygnus X-3. From the widths of the RRC features, electron temperatures of $\sim 5-100 \mathrm{eV}$ are inferred for the X-ray emission-line regions. As predicted, these temperatures are far below the temperatures where these ions are formed in coronal equilibrium.

The upcoming launches of $A X A F, X M M$, Spectrum RöntgenGamma, and Astro $E$ (for a review see Warwick 1997) are expected to open a new era in the spectroscopy of extrasolar $\mathrm{X}$-ray sources. The combination of the large collecting area telescopes and high-resolution spectrometers on these satellites will produce high-quality spectra to which a wide range of plasma diagnostic techniques will be applied. Of particular interest will be the $0.7-2.0 \mathrm{keV}(6-18 \AA$ ) spectral band, which is dominated by the $L$-shell transitions (i.e., $n \geq 3 \rightarrow n=2$ ) of Fe XVII to Fe XXIV (the iron $L$-shell ions). These ions exist over a wide range of physical conditions and are expected to provide many valuable plasma diagnostics.

For gases of cosmic abundance in photoionization equilibrium, $L$-shell iron forms at $\log \xi \sim 1.8-2.8$ (Kallman et al. 1996). The electron temperature in this range of $\xi$ is $\lesssim 100 \mathrm{eV}$. $L$-shell iron overlaps in $\xi$ with $\mathrm{Ne} \mathrm{X}, \mathrm{Mg}$ XII, Si XIV, and $\mathrm{S}$ XVI, which form at $\log \xi \sim 2-2.5$. Thus, the temperatures measured in $4 U$ 1626-67 and Cyg X-3 largely validate the predicted temperature range for $L$-shell iron. However, the exact temperature at which a given ion forms depends on many variables, such as the metallicity of the gas, the shape of the ionizing spectrum, the presence of additional heating and/or cooling mechanisms, and radiative transfer effects. These can be expected to vary for different sources. For most iron $L$-shell ions at these low temperatures, DR is the dominant recombination process in the plasma. It is therefore important that the iron $L$-shell DR rates be benchmarked over a range that encompasses the predicted temperature range.

DR begins when a free electron collisonally excites a bound (core) electron of an ion and is simultaneously captured by the 


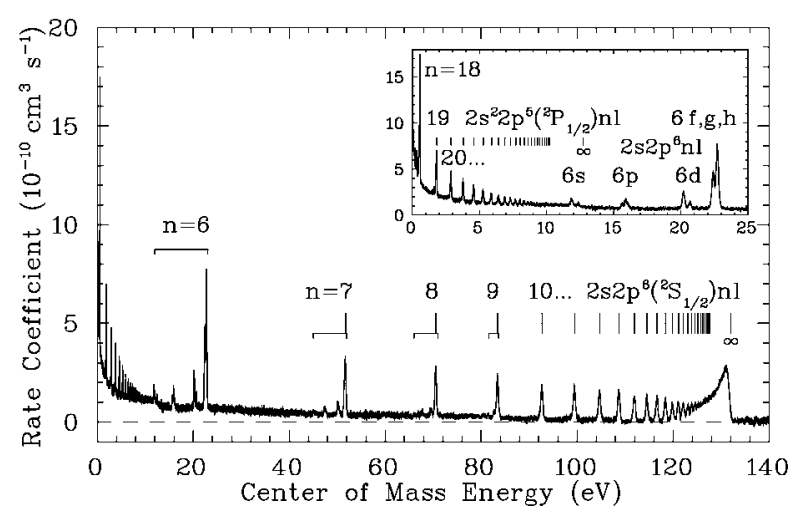

FIG. 1.-Measured Fe XVIII to Fe XVII recombination rate coefficient vs. electron-ion collision energy. DR resonances resulting from ${ }^{2} P_{3 / 2}-{ }^{2} P_{1 / 2}$ and ${ }^{2} P_{3 / 2}-{ }^{2} S_{1 / 2}$ core excitations are labeled. The nonresonant "background" recombination rate is due to $R R$.

ion. DR occurs when the recombined ion radiatively stabilizes (instead of autoionizing) and emits a photon that reduces the total energy of the system to below its ionization limit. For DR to occur, energy conservation requires $E_{k}=\Delta E-E_{b}$, where $E_{k}$ is the kinetic energy of the free electron, $\Delta E$ is the excitation energy of the bound electron, and $E_{b}$ is the binding energy released when the free electron is captured. Because $\Delta E$ and $E_{b}$ are quantized, DR occurs only for specific resonant energies. The resonance structure of DR determines its temperature dependence. For $L$-shell iron, $\Delta n=0 \mathrm{DR}$ occurs only for $E_{k} \lesssim 150 \mathrm{eV}$, and $\Delta n=1 \mathrm{DR}$ occurs only for $E_{k} \gtrsim 250$ $\mathrm{eV}$. As a result, $\Delta n=0 \mathrm{DR}$ is the dominant DR channel for $L$-shell iron in photoionized gases, and $\Delta n=1 \mathrm{DR}$ is the dominant DR channel in collisionally ionized plasmas (e.g., coronal plasmas).

To address the needs for modeling photoionized gases, we have initiated a series of experiments to measure the $\Delta n=0$ DR rates for the iron $L$-shell ions. Measurements are carried out using the heavy-ion test storage ring (TSR) at the MaxPlanck-Institut für Kernphysik in Heidelberg, Germany (Habs et al. 1989). Here we present our results for $\Delta n=0 \mathrm{DR}$ of $\mathrm{Fe}$ XVIII. In particular, we have investigated the capture channels

$$
\begin{aligned}
\mathrm{Fe}^{17+}\left(2 s^{2} 2 p^{5}\left[{ }^{2} P_{3 / 2}\right]\right)+e^{-} \\
\rightarrow \begin{cases}\mathrm{Fe}^{16+}\left(2 s^{2} 2 p^{5}\left[{ }^{2} P_{1 / 2}\right] n l\right) & (n=18, \ldots, \infty) ; \\
\mathrm{Fe}^{16+}\left(2 s 2 p^{6}\left[{ }^{2} S_{1 / 2}\right] n l\right) & (n=6, \ldots, \infty) .\end{cases}
\end{aligned}
$$

The radiative stabilization of these autoionizing states to bound configurations of Fe XVII leads to DR resonances for collision energies between 0 and $132 \mathrm{eV}$.

\section{EXPERIMENTAL TECHNIQUE}

The essence of the experimental technique involves using ions stored in an ion storage ring, merging them with a precision-controlled electron beam in one of the straight sections of the ring, and after the demerging of the two beams, using a dipole magnet to separate the recombined ions from the stored ions and to direct the recombined ions onto a particle counter. The experimental arrangement and measuring technique have been described elsewhere (Kilgus et al. 1992; Lampert et al. 1996; Müller \& Wolf 1997) and are only briefly reviewed here. Negative ions are accelerated and stripped using a tandem accelerator, post-accelerated to $311 \mathrm{MeV}$, stripped to their final charge state, and accumulated in TSR using repeated multiturninjection stacking techniques (Grieser et al. 1991) and electron cooling (Poth 1990) to yield circulating currents of 30-50 $\mu \mathrm{A}$. With cooling, the storage lifetime is $\sim 50 \mathrm{~s}$ and the beam diameter is $\sim 2-3 \mathrm{~mm}$. Data acquisition begins $\sim 6 \mathrm{~s}$ after stacking has ended. This is long compared to the lifetime of metastable $\mathrm{Fe}^{17+}\left(2 s^{2} 2 p^{5}\left[{ }^{2} P_{1 / 2}\right]\right)$ (Cheng, Kim, \& Desclaux 1979), so all the stored ions are in the ground state.

Electron cooling is achieved using a beam of electrons that is guided by a longitudinal magnetic field $(\sim 21 \mathrm{mT})$ and, in one of the straight sections of TSR, merged with the circulating ion beam for a nominal length of $1.5 \mathrm{~m}$. The electron beam velocity is matched to that of the ions. Before merging, the electron beam is adiabatically expanded (from a diameter of 1.9 to $\sim 5.1 \mathrm{~cm}$ ) to reduce the velocity spread of the electrons transverse to their direction of motion (Pastuszka et al. 1996). The velocity spread in the electron-ion center of mass (c.m.) frame is best described by a "flattened" (i.e., nonisotropic) Maxwellian distribution with a transverse temperature $k_{\mathrm{B}} T_{\perp} \approx$ $18 \mathrm{meV}$ and a longitudinal temperature $k_{\mathrm{B}} T_{\|} \approx 0.18 \mathrm{meV}$. During data acquisition the electron beam is precisely modulated so that after expansion, the electron beam density for the present measurements varied between $\sim 1.5$ and $2.5 \times 10^{7} \mathrm{~cm}^{3}$.

The electron cooler also serves as an electron target. For recombination measurements, the electron energy in the c.m. frame is chopped between cooling and measurement energies. Recombined ions are separated from the circulating ion beam by the first dipole magnet downstream of the cooler and are detected using a fast scintillator, heavy-ion detector with a detection efficiency of more than 95\% (Miersch et al. 1996). Recombined ions in which the outer electron is in $n \geq n_{\text {cut }}=$ 55 are expected to be field ionized by the dipole magnet and not detected. However, the distance from the center of the cooler to the dipole magnet is $\sim 5.1 \mathrm{~m}$, during which the captured electrons can radiatively decay to below $n_{\text {cut }}$. Using the hydrogenic formula of Marxer \& Spruch (1991) and the fact that DR populates mostly $l \leq 8$, we estimate that electrons captured into $n \lessgtr 124$ will radiatively decay below $n_{\text {cut }}$ and that DR via capture into these levels is detected in our experimental arrangement.

The measured recombination rate coefficient $\alpha_{L}$ is given by $\alpha_{L}(E)=R \gamma^{2} /\left(n_{e} N_{i} L / C\right)$. Here $L$ is the nominal length of the electron-ion overlap in the electron cooler, $E$ is the c.m. energy, $R$ is the recombination signal rate, $n_{e}$ is the electron density, $N_{i}$ is the number of stored ions in the ring, $C=55.4 \mathrm{~m}$ is the circumference of TSR, and $\gamma^{2}=\left[1-\left(v_{i} / c\right)^{2}\right]^{-1} \approx 1.01$, where $v_{i}$ is the ion velocity and $c$ is the speed of light. The total measurement uncertainty is estimated to be better than $\pm 20 \%$ (at a confidence level believed to be equivalent to a $90 \%$ statistical confidence level). The dominant sources of uncertainty stem from the ion current measurement, the electron beam expansion factor, which affects the electron density determination, and the recombined ion detection efficiency.

\section{RESULTS AND DISCUSSION}

The measured Fe XVIII to Fe XVII recombination rate coefficient versus electron-ion collision energy is shown in Figure 1. Clearly visible are the ${ }^{2} P_{3 / 2}-{ }^{2} P_{1 / 2}$ resonance series due to finestructure core excitations at low energies and the ${ }^{2} P_{3 / 2}-{ }^{2} S_{1 / 2}$ resonance series at higher energies, similar to earlier measurements on the isoelectronic Se XXVI (Lampert et al. 1996). The effects of merging and demerging the electron and ion beams are corrected for, as described by Lampert et al. (1996). The 


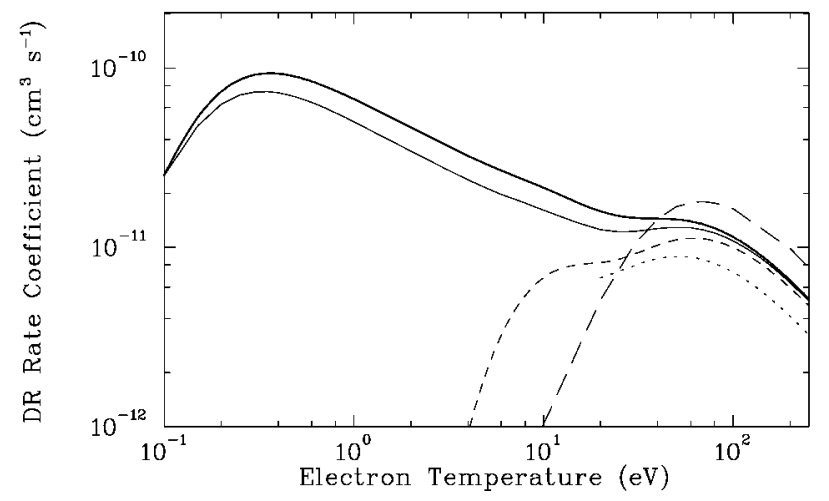

FIG. 2.-Fe XVIII to Fe XVII Maxwellian-averaged DR rate coefficients. The solid thick line is the integration of the experimental DR resonance strengths and energies extracted from the results shown in Fig. 1. Existing calculations by Roszman (1987) (long-dashed line), Chen (1988) (dotted line), and Dasgupta \& Whitney (1990) (short-dashed line) do not include the ${ }^{2} P_{3 / 2}-{ }^{2} P_{1 / 2}$ DR channel. The thin solid line shows our calculations, including this channel.

measured DR signal appears on top of a nonresonant "background" due to RR. Recombination due to charge transfer off residual gas in TSR is taken into account by subtracting a constant such that the background level at $134 \mathrm{eV}$ matches the very low theoretical RR rate at that energy (Lampert et al. 1996). The c.m. energy scale has been reduced by a factor of $\sim 1.02$ so our experimentally determined DR series limits (see Kilgus et al. 1992) match the spectroscopically measured energies of the Fe XVIII $2 s^{2} 2 p^{5}\left({ }^{2} P_{3 / 2}\right) \rightarrow 2 s^{2} 2 p^{5}\left({ }^{2} P_{1 / 2}\right)$ and $2 s^{2} 2 p^{5}\left({ }^{2} P_{3 / 2}\right) \rightarrow 2 s 2 p^{6}\left({ }^{2} S_{1 / 2}\right)$ transitions (Shirai et al. 1990). Individual DR resonance strengths were determined as described by Kilgus et al. (1992). A detailed presentation of the individually measured resonance strengths will be given elsewhere (Savin et al. 1997, in preparation).

In Figure 2, the measured DR resonance strengths and energies have been integrated with an isotropic Maxwellian electron velocity distribution to yield a total Fe XVIII $\Delta n=0$ DR rate coefficient as a function of electron temperature. We have fitted our inferred DR rates using the formula of AR92:

$$
\alpha_{\mathrm{DR}}\left(T_{e}\right)=T_{e}^{-3 / 2} \sum_{i} c_{i} e^{-E_{i} / k_{\mathrm{B}} T_{e}}
$$

where $T_{e}$ is the electron temperature, $k_{\mathrm{B}}$ is the Boltzmann constant, and $c_{i}$ and $E_{i}$ are the strength parameter and the energy parameter for the $i$ th fitting function component, respectively. The best-fit values are listed in Table 1. The fit for DR via a ${ }^{2} P_{3 / 2}-{ }^{2} P_{1 / 2}$ core excitation is accurate to better than $1.3 \%$ for 0.05 $\mathrm{eV} \leq k_{\mathrm{B}} T_{e} \leq 10^{4} \mathrm{eV}$. Below $0.05 \mathrm{eV}$, the fit rate goes to zero faster than the measured rate, but because the RR rate at these energies is $\gtrsim 600$ times larger than the ${ }^{2} P_{3 / 2}-{ }^{2} P_{1 / 2}$ DR rate, this has an insignificant effect on ionization balance calculations. The fit for DR via a ${ }^{2} P_{3 / 2}-{ }^{2} S_{1 / 2}$ core excitation is accurate to better than $1 \%$ for $5 \mathrm{eV} \leq k_{\mathrm{B}} T_{e} \leq 10^{4} \mathrm{eV}$ and to better than $3.5 \%$ for $4 \mathrm{eV} \leq k_{\mathrm{B}} T_{e} \leq 5 \mathrm{eV}$. Below $4 \mathrm{eV}$, the fit rate goes to zero faster than the measured rate; but because the RR rate at these energies is $\gtrsim 60$ times larger than the ${ }^{2} P_{3 / 2}-{ }^{2} S_{1 / 2}$ DR rate, this also has an insignificant effect on ionization balance calculations. The uncertainty in the absolute magnitude of the fitted rate coefficient is $\pm 20 \%$, which represents our estimated total experimental uncertainty. DR from the unmeasured $n>$ 124 levels are estimated to contribute $\lesssim 2 \%$ to the total DR rate. Using the Inglis-Teller limit (Inglis \& Teller 1939) and
TABLE 1

Fe XVIII to Fe XVII $\Delta N=$ o DR Rate Coefficient Fit PARAMETERS FOR DR VIA THE ${ }^{2} P_{3 / 2}-{ }^{2} P_{\mathrm{I} / 2}$ AND ${ }^{2} P_{3 / 2}-{ }^{2} S_{\mathrm{I} / 2}$ CORE EXCITATIONS

\begin{tabular}{|c|c|c|c|c|c|}
\hline \multirow{2}{*}{\multicolumn{2}{|c|}{$i$}} & \multicolumn{2}{|c|}{${ }^{2} P_{3 / 2}-{ }^{2} P_{1 / 2}$} & \multicolumn{2}{|c|}{${ }^{2} P_{3 / 2}-{ }^{2} S_{1 / 2}$} \\
\hline & & $\begin{array}{c}c_{i} \\
\left(\mathrm{~cm}^{3} \mathrm{~s}^{-1} \mathrm{~K}^{1.5}\right)\end{array}$ & $\begin{array}{c}E_{i} \\
(\mathrm{eV})\end{array}$ & $\begin{array}{c}c_{i} \\
\left(\mathrm{~cm}^{3} \mathrm{~s}^{-1} \mathrm{~K}^{1.5}\right)\end{array}$ & $\begin{array}{c}E_{i} \\
(\mathrm{eV})\end{array}$ \\
\hline 1 & & $5.50(-6)$ & $2.29(-1)$ & $1.73(-3)$ & $19.1(0)$ \\
\hline 2 & & $9.56(-5)$ & $5.38(-1)$ & $3.72(-3)$ & $50.6(0)$ \\
\hline 3 & & $9.78(-5)$ & $1.71(0)$ & $3.23(-2)$ & $120 .(0)$ \\
\hline 4 & 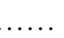 & $2.61(-4)$ & $3.78(0)$ & 0 & 0 \\
\hline 5 & & $5.24(-4)$ & $9.08(0)$ & 0 & 0 \\
\hline
\end{tabular}

our experimental $n_{\text {cut }} \sim 124$, we estimate that our fitted rate coefficient is valid for plasma densities $\$ 3.6 \times 10^{7} \mathrm{~cm}^{-3}$.

Various theoretical Fe XVIII $\Delta n=0$ DR rates are also shown in Figure 2. At $k_{\mathrm{B}} T_{e} \sim 15 \mathrm{eV}$, near where Fe XVIII is predicted to peak in fractional abundance in photoionized gases, our inferred DR rate is a factor of $\sim 2$ larger than the calculations of Roszman (1987), Chen (1988), and Dasgupta \& Whitney (1990). These theoretical rates all tend rapidly to zero at $k_{\mathrm{B}} T_{e} \lesssim 20 \mathrm{eV}$ because they have not included ${ }^{2} P_{3 / 2}-{ }^{2} P_{1 / 2}$ DR. The calculations of Roszman (1987) and Dasgupta \& Whitney (1990) used $L S$ coupling and thus do not include fine-structure core excitations. The calculations of Chen (1988) used intermediate coupling, which can account for fine structure. But Chen did not include the $2 p_{1 / 2} \rightarrow 2 p_{3 / 2}$ channel because the calculations were carried out for the high temperatures of collisionally ionized plasmas (where this channel is unimportant). Related work has been carried out in $L S$ coupling, which does not account for DR via fine-structure core excitations but which does include the effects of fine-structure autoionizing transitions of the intermediate state of the DR process (see LaGattuta, Nasser, \& Hahn 1986; Ramadan \& Hahn 1989).

Also shown in Figure 2 are our new fully relativistic calculations, using the multiconfiguration Dirac-Fock method in intermediate coupling with configuration interaction within the same complex. Details of these calculations, extending earlier work by Chen (1988), will be given in Savin et al. (1997, in preparation). The ${ }^{2} P_{3 / 2}-{ }^{2} P_{1 / 2}$ channel is now included. DR is calculated accounting for both immediate radiative stabilization and a single cascade between autoionizing states followed by radiative stabilization. Thus, for the ${ }^{2} P_{3 / 2}-{ }^{2} S_{1 / 2}$ channel, autoionization via a $2 p_{3 / 2}-2 p_{1 / 2}$ transition (which can occur for capture into $n \geq 18$ ) is also included. Calculations were carried out for $n \leq 36$ and $l \leq 8$ and the results extrapolated for $n>36$ and $l>8$. Extrapolations for $l>8$ were calculated using a power law fitted to $l=6,7$, and 8. Extrapolations for $n>36$ were carried out using an $n^{-3}$ scaling for the Auger and radiative rates. These new calculations agree to within $\lesssim 30 \%$ with the measurements.

\section{ASTROPHYSICAL IMPLICATIONS}

Commonly used models of photoionized gases are XSTAR (Kallman \& Krolik 1997), ION (Netzer 1993), and CLOUDY (Ferland 1996). These models use the recommended DR rates of AR92 for iron. For Fe XVIII, AR92 incorporate the rates of Dasgupta \& Whitney (1990). Our inferred rates are larger than those of Dasgupta \& Whitney, by as much as a factor of $\sim 2$ at $k_{\mathrm{B}} T_{e} \sim 15 \mathrm{eV}$. Implementing our rates into the above models will shift the Fe XVIII peak fractional abundance to higher ionization parameters. The magnitude of this effect remains to be determined and will require corrections to the low-temper- 
ature DR rates for all relevant charge states that contribute to the heating and cooling of the gas.

For the other cosmically abundant elements, XSTAR uses the recommended DR rates of Aldrovandi \& Péquignot (1973), ION uses the recommended rates of Shull \& Van Steenberg (1982), and CLOUDY uses the recommended rates of AR85 supplemented by the $L S$-coupling calculations by Nussbaumer \& Storey $(1983,1984,1986,1987)$ of DR at nebular temperatures. These recommended rates (and many of the recommended rates of AR92) are calculated using either the Burgess (1965) formula or $L S$ coupling. The Burgess formula is known to be inappropriate for low temperatures (Storey 1983). And, as we have shown, $L S$-coupling calculations do not properly account for all possible DR channels at nebular temperatures, especially for $L$-shell ions where DR can proceed via finestructure core excitations. Similar problems may exist for $M$ shell ions, where DR can proceed via $3 l_{j} \rightarrow 3 l_{j^{\prime}}$ core excitations. A reevaluation of the recommended low-temperature DR rates is needed to assess for what ions new rates must be determined.

The temperature dependence of Fe XVIII $\Delta n=0$ DR has a number of spectroscopic implications. At $k_{\mathrm{B}} T_{e} \lesssim 15 \mathrm{eV}$ the dominant DR rate is via the ${ }^{2} P_{3 / 2}-{ }^{2} P_{1 / 2}$ core excitation. Because the radiative transition of the inner electron is electric-dipole forbidden, the resulting autoionizing states of Fe XVII stabilize by radiative decay of the captured electron. $\Delta E$ for the Fe XVIII ${ }^{2} P_{3 / 2}-{ }^{2} P_{1 / 2}$ transition is $12.72 \mathrm{eV}$ (Shirai et al. 1990). Using the hydrogenic approximation for $E_{b}$, we find that the recombining electrons are captured into levels $n \geq 18$. Accurately calculating the resulting, cascade-generated X-ray spectrum promises to be theoretically challenging. A similar situation can probably be expected for any ion that undergoes DR via forbidden, finestructure transitions.

Lines produced by $\Delta n=0 \mathrm{DR}$ also offer the possibility of a new temperature diagnostic (Liedahl 1992, Kahn \& Liedahl
1995). Fe XVIII ${ }^{2} P_{3 / 2}-{ }^{2} P_{1 / 2}$ DR can generate Fe XVII $2 s^{2} 2 p^{6}-2 s^{2} 2 p^{5}\left({ }^{2} P_{1 / 2}\right) 3 l$ lines. Fe XVIII ${ }^{2} P_{3 / 2}-{ }^{2} S_{1 / 2}$ DR will primarily stabilize via a $2 p \rightarrow 2 s$ radiative decay but can also stabilize by the radiative decay of the captured electron. As the captured electron cascades down, it can generate $\mathrm{Fe}$ XVII $2 s^{2} 2 p^{6}-2 s 2 p^{6}\left({ }^{2} S_{1 / 2}\right) 3 l$ lines. Both of these transitions involve an excited core configuration. RR, however, does not produce an excited core, and it will result in Fe XVII $2 s^{2} 2 p^{6}-2 s^{2} 2 p^{5}\left({ }^{2} P_{3 / 2}\right) 3 l$ lines. It is worth noting that because of their temperature behavior, the ${ }^{2} P_{3 / 2}-{ }^{2} P_{1 / 2}$ DR and the ${ }^{2} P_{3 / 2}-{ }^{2} S_{1 / 2}$ DR are emitted by different temperature regions of a plasma. Because ${ }^{2} P_{3 / 2}-{ }^{2} P_{1 / 2} \mathrm{DR},{ }^{2} P_{3 / 2}-{ }^{2} S_{1 / 2} \mathrm{DR}$, and RR all have distinctly different temperature dependences, ratios formed from lines produced by these three processes may provide temperature diagnostics. Similar mechanisms should exist for any $L$-shell ion. Further experimental and theoretical work is in progress to explore this new class of temperature diagnostics.

We thank M. Grieser, R. Repnow, and the TSR group for their expert technical support during the beam time. The authors thank T. R. Kallman, F. Paerels, J. C. Raymond, and D. A. Verner for stimulating conversations. This work was supported in part by NASA High Energy Astrophysics X-Ray Astronomy Research and Analysis grant NAGW-4185. Travel to Heidelberg and living expenses for D. W. S. were supported by NATO Collaborative Research grant CRG-950911. The experimental work has been supported in part by the German Federal Minister for Education, Science, Research, and Technology (BMBF) under contracts 06 GI 475, 06 GI 848, and 06 HD 854I. Work performed at Lawrence Livermore National Laboratory was performed under the auspices of the US Department of Energy (contract W-7405-ENG-48).

\section{REFERENCES}

Aldrovandi, S. M. V., \& Péquignot, D. 1973, A\&A, 25, 137; erratum 47, 321 (1976)

Angelini, L., White, N. E., Nagase, F., Kallman, T. R., Yoshida, A., Takeshima, Becker, C., \& Paerels, F. 1995, ApJ, 449, L41

Arnaud, M., \& Raymond, J. 1992, ApJ, 398, 394 (AR92)

Arnaud, M., \& Rothenflug, R. 1985, A\&AS, 60, 425 (AR85)

Burgess, A. 1965, ApJ, 141, 1588

Chen, M. H. 1988, Phys. Rev. A, 38, 2332

Cheng, K. T., Kim, Y.-K., \& Desclaux, J. P. 1979, At. Data Nucl. Data Tables, 24, 111

Dasgupta, A., \& Whitney, K. G. 1990, Phys. Rev. A, 42, 2640

Ferland, G. H. 1996, Hazy, A Brief Introduction to Cloudy, Univ. Kentucky Int. Rep.

Grieser, M., et al. 1991, in Cooler Rings and Their Applications, ed. T. Katayama \& A. Noda (Singapore: World Scientific)

Habs, D., et al. 1989, Nucl. Instr. Methods, B43, 390

Hatchett, S., Buff, J., \& McCray, R. 1976, ApJ, 206, 847

Inglis, D. R., \& Teller, E. 1939, ApJ, 90, 439

Kahn, S. M., \& Liedahl, D. A. 1995, in Physics of Multiply Charged Ions, ed. D. Liesen (New York: Plenum), 169

Kallman, T. R., \& Krolick, J. H. 1997, XTSAR, A Spectral Analysis Tool

Kallman, T. R., Liedahl, D., Osterheld, A., Goldstein, W., \& Kahn, S. 1996, ApJ, 465, 994

Kallman, T. R. \& McCray, R. 1982, ApJS, 50, 263

Kilgus, G., Habs, D., Schwalm, D., Wolf, A., Badnell, N. R., \& Müller, A. 1992, Phys. Rev. A, 46, 5730

LaGattuta, K., Nasser, I., \& Hahn, Y. 1986, Phys. Rev. A, 33, 2782

Lampert, A., Wolf, A., Habs, D., Kilgus, G., Schwalm, D., Pindzola, M. S., \& Badnell, N. R. 1996, Phys. Rev. A, 53, 1413
Liedahl, D. A. 1992, Ph.D. thesis, Univ. California, Berkeley

Liedahl, D. A., Kahn, S. M., Osterheld, A. L., \& Goldstein, W. H. 1990, ApJ, 350, L37

Liedahl, D. A., \& Paerels, F. 1996, ApJ, 468, L33

Marxer, H., \& Spruch, L. 1991, Phys. Rev. A, 43, 1268

Miersch, G., Habs, D., Kenntner, J., Schwalm, D., \& Wolf, A. 1996, Nucl. Instr. Methods A, 369, 277

Müller, A., \& Wolf, A. 1997, in Accelerator-Based Atomic Physics Techniques and Applications, ed. J. C. Austin \& S. M. Shafroth (New York: AIP), in press

Netzer, H. 1993, ApJ, 411, 594

Nussbaumer, H., \& Storey, P. J. 1983, A\&A, 126, 75

. 1984, A\&AS, 56, 293

- 1986, A\&AS, 64, 545

1987, A\&AS, 69, 123

Pastuszka, S., et al. 1996, Nucl. Instr. Methods A, 369, 11

Poth, H. 1990, Phys. Rep., 196, 135

Ramadan, H. H., \& Hahn, Y. 1989, Phys. Rev. A, 39, 3350

Roszman, L. J. 1987, Phys. Rev. A, 35, 2138

Shirai, T., Funatake, Y., Mori, K., Sugar, J., Wiese, W. L., \& Nakai, Y. 1990 J. Phys. Chem. Ref. Data, 19, 127

Shull, J. M., \& Van Steenberg, M. 1982, ApJS, 48, 95; 49, 351

Storey, P. J. 1983, in Planetary Nebulae, ed. D. R. Flower (Dordrecht: Reidel), 199

Tanaka, Y., Inoue, H., \& Holt, S. M. 1994, PASJ, 46, L37

Tarter, C. B., Tucker, W. H., \& Salpeter, E. E. 1969, ApJ, 156, 943

Warwick, R. S. 1997, in Workshop Proc. Leicester X-Ray Astronomy Group Spec. Rep. XRA97/02, The Next Generation of X-Ray Observatories, ed. M. J. L. Turner \& M. G. Watson (Leicester: Univ. Leicester), 7 